\section{Pieloplastia laparoscópica asistida por robot en un paciente monorreno con estenosis ureteropiélica}

Viana-Álvarez G, ${ }^{1}$ Villeda-Sandoval Cl, ${ }^{2}$ Orozco-Lara Juan C, ${ }^{2}$ Trujillo-Ortiz L, ${ }^{1}$ Sánchez-Aquino $U,{ }^{1}$ y colaboradores.

\section{Resumen}

ANTECEDENTES: la estenosis de la unión ureteropiélica es la causa más frecuente de hidronefrosis en pacientes pediátricos y en adultos jóvenes. Esta alteración se caracteriza por obstrucción anatómica o funcional del flujo de orina, de la pelvis renal al uréter, la falta de tratamiento se refleja en deterioro de la función renal.

CASO CLÍNICO: paciente femenina de 22 años de edad, que inició con infección urinaria de repetición y mala reacción al tratamiento dos años antes de efectuar el procedimiento quirúrgico, seguido de dolor lumbar izquierdo en los últimos 6 meses. La tomografía computada reportó hipoplasia renal derecha e hidronefrosis izquierda, por lo que se colocó catéter JJ izquierdo; se realizó pieloplastia laparoscópica asistida por robot, sin complicaciones ni incidentes adicionales. La paciente tuvo adecuada evolución y egresó dos días después del posoperatorio.

CONCLUSIONES: la cirugía robótica disminuye la mayor parte de las complicaciones provocadas por la laparoscopia convencional, debido a la precisión de los movimientos, facilidad en la sutura y visualización en tercera dimensión; además, es efectiva cuando la pieloplastia desmembrada convencional no es óptima o se torna técnicamente complicada.

PALABRAS CLAVE: pielopastia laparoscópica, robot, estenosis ureteral.

Rev Mex Urol. 2017 Jul-Aug;77(4):289-295.

\section{Robotic-assisted laparoscopic pyeloplasty in a patient with one functioning kidney with ureteropelvic junction obstruction}

Viana-Álvarez G, ${ }^{1}$ Villeda-Sandoval CI, ${ }^{2}$ Orozco-Lara Juan C, ${ }^{2}$ Trujillo-Ortiz L, ${ }^{1}$ Sánchez-Aquino $U,{ }^{1}$ et al.

\section{Abstract}

BACKGROUND: Narrowness of the ureteropelvic junction is the most frequent cause of hydronephrosis in young adults and children. It is
${ }^{1}$ División de Urología, Hospital General Dr. Manuel Gea González, Ciudad de México.

${ }^{2}$ División de Urología, Hospital Naval General de Alta Especialidad, Ciudad de México.

Recibido: diciembre 2016

Aceptado: mayo 2017

Correspondencia

Dr. Guillermo Viana Álvarez

guillermo_nec@hotmail.com

Este artículo debe citarse como

Viana-Álvarez G, Villeda-Sandoval Cl, Orozco-Lara Juan C, y col. Pieloplastia laparoscópica asistida por robot en un paciente monorreno con estenosis ureteropiélica. Rev Mex Urol. 2017 jul-agos;77(4):289-295. DOI: https://doi.org/10.24245/revmexurol.v77i4.1090 
characterized by anatomic or functional obstruction of the urine flow from the renal pelvis to the ureter that, when untreated, conditions impaired kidney function.

CLINICAL CASE: A 22-year-old woman had recurrent urinary infection with poor treatment response for the past 2 years and left lumbar pain of 6-month progression. Computed tomography revealed right renal hypoplasia and left hydronephrosis, for which a left double-J catheter was placed. Robotic-assisted laparoscopic pyeloplasty was performed with no complications or incidents. The patient had adequate progression and was released from the hospital two days later.

CONCLUSIONS: Robotic-assisted surgery mitigates many of the complications resulting from conventional laparoscopy thanks to its movement precision, suturing ease, and three-dimensional vision. It is also very useful when standard dismembered pyeloplasty may be sub-optimal or technically complicated.

KEYWORDS: Laparoscopic pyeloplasty; Robot; Ureteral stricture

\author{
${ }^{1}$ Urology Division, Hospital General Dr. Ma- \\ nuel Gea González, Mexico City. \\ ${ }^{2}$ Urology Division, Hospital Naval General \\ de Alta Especialidad, Mexico City. \\ Correspondence \\ Dr. Guillermo Viana Álvarez \\ guillermo_nec@hotmail.com
}

\section{ANTECEDENTES}

La estenosis de la unión pieloureteral es la alteración congénita más frecuente de la vía urinaria superior. Suele diagnosticarse en la adolescencia o en la edad adulta, pues en estas etapas comienza a manifestarse clínicamente. La causa más común es la alteración congénita de la unión pieloureteral; sin embargo, se han descrito causas de origen ureteral (válvulas ureterales, anomalías en la inserción del uréter) y extraureteral (adherencias, fibrosis, vasos anómalos). ${ }^{1}$ Aproximadamente 1 de cada 20,000 recién nacidos manifiesta esta enfermedad. ${ }^{2}$

La pieloplastia abierta es el tratamiento de elección de la estenosis ureteropiélica, con tasas de éxito de 90 a 100\%; no obstante, a pesar de los buenos resultados, la morbilidad y el tiempo de recuperación elevados han llevado a la búsqueda de técnicas adicionales mínimamente invasivas.
En 1993 Lucas describió la primera pieloplastia laparoscópica como una técnica segura, que disminuye el tiempo de recuperación, la morbilidad y su tasa de éxito es similar a la técnica abierta. ${ }^{3}$ Sus principales desventajas incluyen: requerimiento de material adecuado y adiestramiento en técnicas laparoscópicas, tiempo quirúrgico elevado y no se encuentra disponible en todos los hospitales. Globalmente, supone mayor morbilidad y hospitalización prolongada comparada con las técnicas endoscópicas, pero elimina el riesgo de sangrado por lesión de vasos localizados cerca de la unión pieloureteral. ${ }^{4}$

Entre las técnicas endoscópicas, las primeras en desarrollarse fueron la pielotomía anterógrada y retrógrada. La pielotomía puede realizarse en corte frío, con energía monopolar o con láser. En general, las técnicas endoscópicas ofrecen mejores resultados en pacientes con estenosis ureteropiélica secundaria versus primaria. La principal 
complicación es la posibilidad de lesión vascular y entre sus ventajas sobresalen: escasa morbilidad y es posible realizarla de forma ambulatoria; sin embargo, se ha observado elevada tasa de morbilidad, alto riesgo de sangrado y tasa de éxito de $60-80 \%{ }^{2}$

Con el advenimiento de la cirugía robótica se inició el tratamiento de esta alteración en el año 2002, con una pieloplastia desmembrada tipo Anderson-Hynes, efectuada por Gettman y su equipo de trabajo. ${ }^{5}$ Esta técnica proporciona al operador una visión aumentada en tres dimensiones y mayor movilidad, con más grados de libertad, lo que facilita la sutura interna y ofrece al cirujano una ergonomía superior; además, la curva de aprendizaje es menor a la de la cirugía laparoscópica. ${ }^{5,6}$

El objetivo de este estudio es reportar un caso de pieloplastia laparoscópica asistida por robot, en un paciente monorreno con estenosis ureteropiélica.

\section{CASO CLÍNICO}

Mujer de 22 años de edad, sin antecedentes de importancia para el padecimiento actual, quien inició 2 años previos a la intervención quirúrgica con cuadros repetidos de infección de vías urinarias, con mala reacción al tratamiento, seguido de dolor lumbar izquierdo tipo cólico 6 meses antes de la cirugía, caracterizado por intensidad de 7/10, con irradiación a la fosa iliaca izquierda y los genitales, y disminución parcial del cuadro con la administración de antinflamatorios no esteroides (AINES). Como estudio inicial se solicitó tomografia axial computada, que evidenció hipoplasia renal derecha e hidronefrosis izquierda, con adelgazamiento del uréter en el tercio superior, en fase de eliminación y retraso en la excreción del medio de contraste. Un mes previo al tratamiento quirúrgico se le colocó un catéter J izquierdo; sin embargo, se decidió tratar la alteración mediante pieloplastia desmembrada laparoscópica asistida por robot.

La técnica quirúrgica se efectúa de la siguiente manera: se posiciona al paciente en decúbito lateral derecho, se colocan 6 trócares con visión directa, 3 trócares robóticos de $5 \mathrm{~mm}$ y 2 convencionales de $12 \mathrm{~mm}$ y 1 de $5 \mathrm{~mm}$ (Figura 1). Se inicia el montaje (docking) del equipo DaVinci Si, tiempo de consola con descenso de colon hasta identificar el uréter izquierdo, con disección en dirección cefálica (Figura 2) hasta identificar la pelvis dilatada. Se realiza el corte del uréter en la unión ureteropiélica, del que se extrae el catéter JJ previamente colocado (Figura 3); posteriormente se procede al corte de pelvis excedente con tijera laparoscópica (Figura 4), que se extrae por el trocar del asistente y se inicia la plastia pélvica con Vicryl 2-0 y sutura continua (Figura 5); el extremo ureteral se espatula (Figura 6) y se inicia la anastomosis ureteropiélica (Figura 7). Mediante aguja de Chiba se introduce la guía hidrofílica dirigida al extremo ureteral, con posterior introducción del catéter JJ 24 por $6 \mathrm{Fr}$, (Figura 8) se dirige el rizo superior en la pelvis y se procede a completar la anastomosis con sutu-

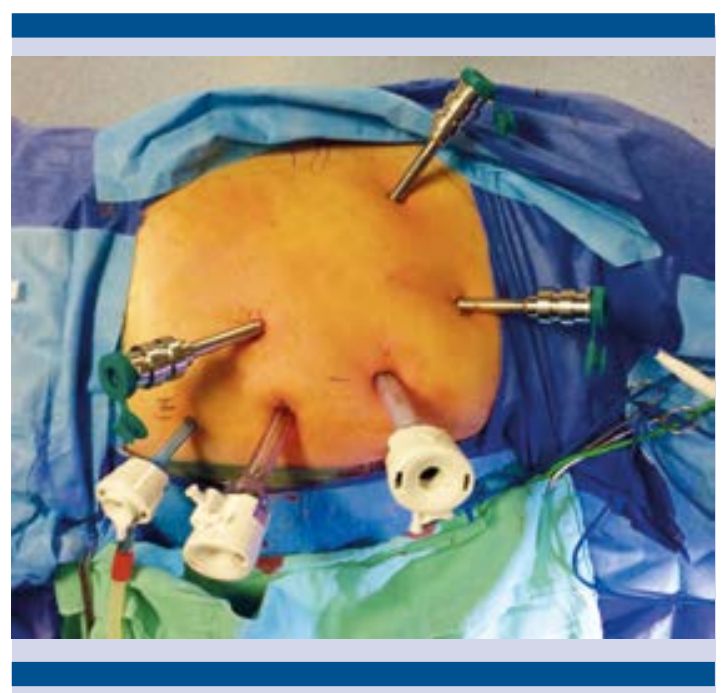

Figura 1. Localización de los trócares. 


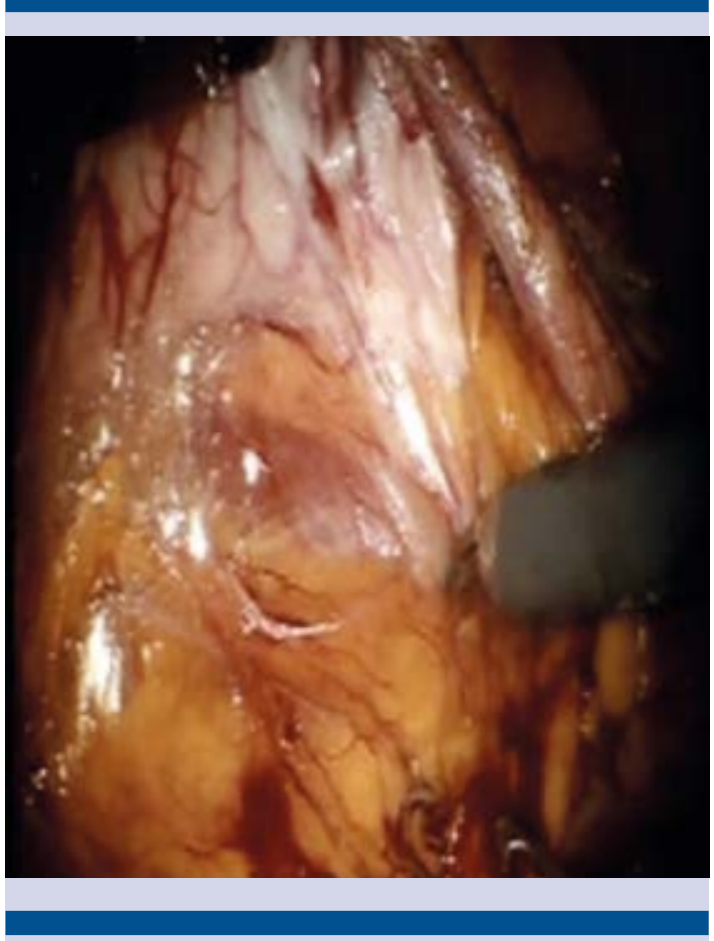

Figura 2. Disección de la pelvis y el uréter.

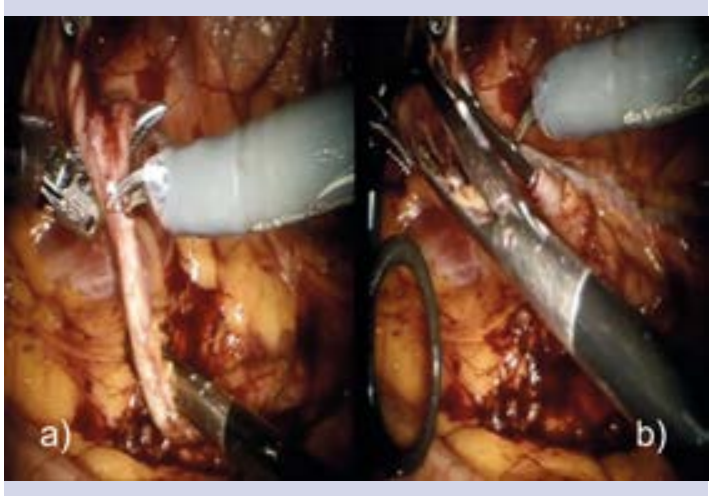

Figura 3. Apertura ureteral (A) y extracción del catéter JJ (B).

ra continua y Vicryl 2-0 (Figura 9); se introduce drenaje Biovack por el trocar del asistente y se finaliza el procedimiento.

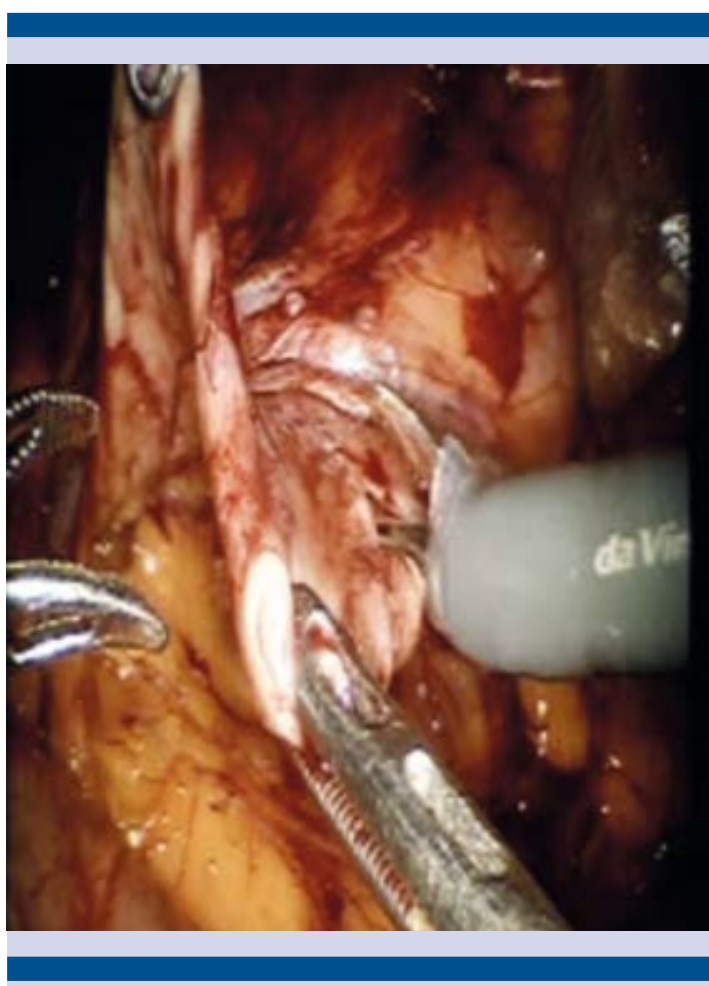

Figura 4. Resección del excedente de pelvis renal.

Después del procedimiento no se registraron complicaciones ni incidentes, el tiempo quirúrgico total fue de 140 minutos y el sangrado estimado de $50 \mathrm{~mL}$. La paciente tuvo adecuada evolución posquirúrgica, con egreso hospitalario dos días después de la cirugía.

\section{DISCUSIÓN}

La introducción de la tecnología robótica, para fines de cirugía reconstructiva compleja, disminuye algunas de las limitaciones asociadas con la laparoscopia, pues ofrece una visión tridimensional amplia, elimina el temblor y aumenta la comodidad del cirujano, además del rango de movimiento de la muñeca, imitando la mano.?

La pieloplastia laparoscópica es una técnica equiparable con la pieloplastia abierta, con la ventaja de ser menos invasiva, más eficiente y 


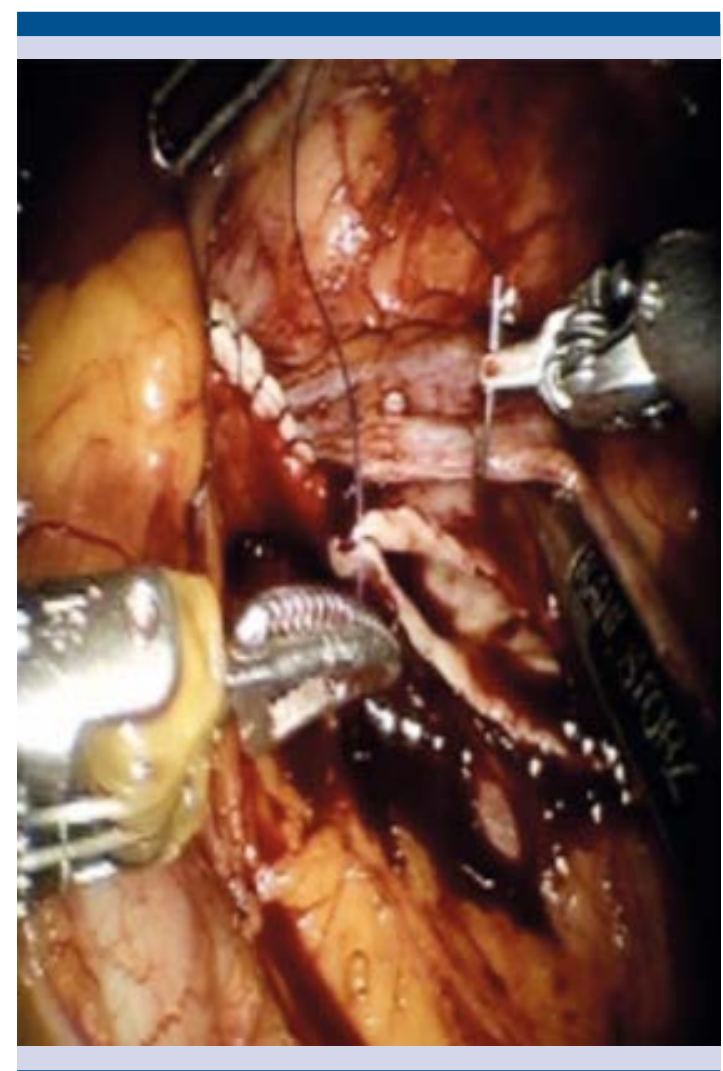

Figura 5. Plastia posterior de la resección de pelvis.

eficaz, y estancia hospitalaria más corta. Es probable que la pieloplastia robótica surja como el nuevo procedimiento quirúrgico de referencia mínimamente invasivo, siempre y cuando se encuentre disponible en la mayor parte de los centros hospitalarios, debido a su precisión y menor curva de aprendizaje; ${ }^{8}$ sin embargo, una de sus desventajas es el costo elevado, pues se ha estimado un aumento aproximado de $\$ 3500$ dólares por paciente, esperando que disminuya conforme se difunda la cirugía robótica. ${ }^{9}$

Hoy día, el centro con mayor experiencia en pieloplastia robótica se encuentra en Austria, encabezado por Schwentner y su grupo. ${ }^{10}$ En un estudio previo, estos autores informaron una tasa de éxito de $96.7 \%$ en 92 pacientes (80 reparaciones primarias y 12 secundarias), con media de seguimiento

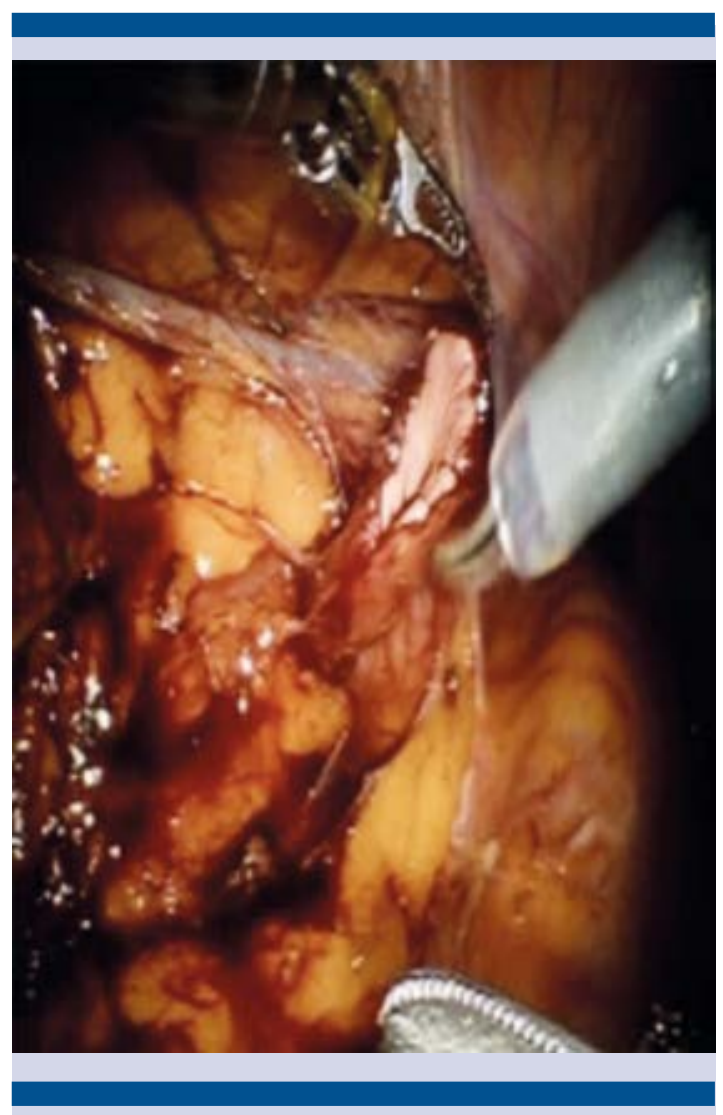

Figura 6. Espatulación ureteral.

de 39.1 meses. El promedio de tiempo quirúrgico fue de 108.3 minutos, estancia hospitalaria de 4-6 días y sangrado operatorio mínimo.

A pesar de los excelentes resultados informados con la pieloplastia asistida por robot, existen desventajas inherentes al uso de esta tecnología. La pérdida de retroalimentación táctil puede representar un problema para el cirujano que está iniciando su experiencia. Esto puede ser especialmente complejo en procedimientos que requieren una delicada manipulación de los tejidos, incluso en las intervenciones que se realizan en un campo operado anteriormente, por ejemplo: en la reparación de estenosis ureteropiélica secundaria. ${ }^{10}$ Una preocupación adicional es la necesidad de un asistente con experiencia, en algunas ocasiones, para ayudar a simplificar la 


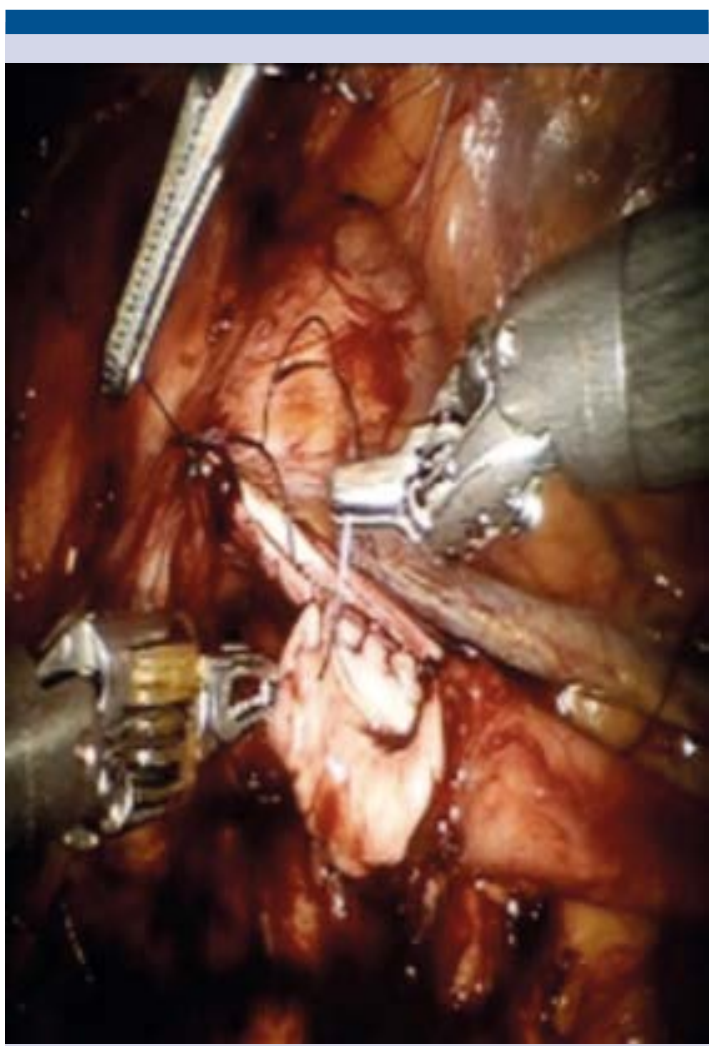

Figura 7. Anastomosis ureteropiélica.

operación, especialmente al comienzo de la curva de aprendizaje. Otra desventaja es la dificultad de realizar una conversión a cirugía abierta después de identificar una lesión vascular mayor, sobre todo cuando el cirujano principal manipula la consola y se encuentra sin vestimenta quirúrgica estéril. ${ }^{11}$

La laparoscopia asistida por robot es una técnica efectiva en el campo de la urología; sin embargo, el principal inconveniente en esta área es su poca accesibilidad, debido al costo elevado y aplicación primordialmente en el ámbito oncológico.

La reparación de la estenosis ureteropiélica mediante procedimientos laparoscópico y abierto

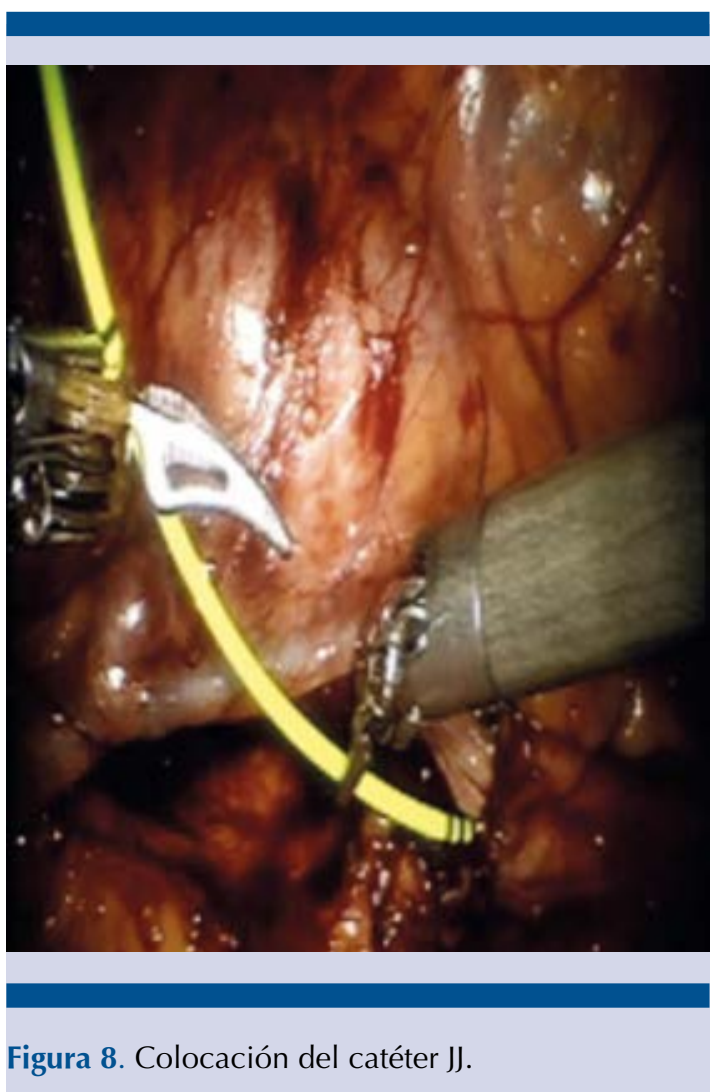

es efectiva y se compara, en muchos aspectos, con las ventajas que ofrece la cirugía de mínima invasión, sobre todo disminución de la morbilidad y menor estancia hospitalaria; sin embargo, el tiempo quirúrgico es más prolongado, pero este factor ha disminuido gracias a la cirugía robótica. También se ha reportado disminución de las complicaciones ocurridas con la laparoscopia convencional, debido a la precisión de los movimientos, facilidad en la sutura y visualización en tercera dimensión, además de ser efectiva cuando la pieloplastia desmembrada de referencia no es óptima o resulta técnicamente complicada.

\section{Financiamiento}

Los autores no recibieron ningún patrocinio para llevar a cabo este estudio. 


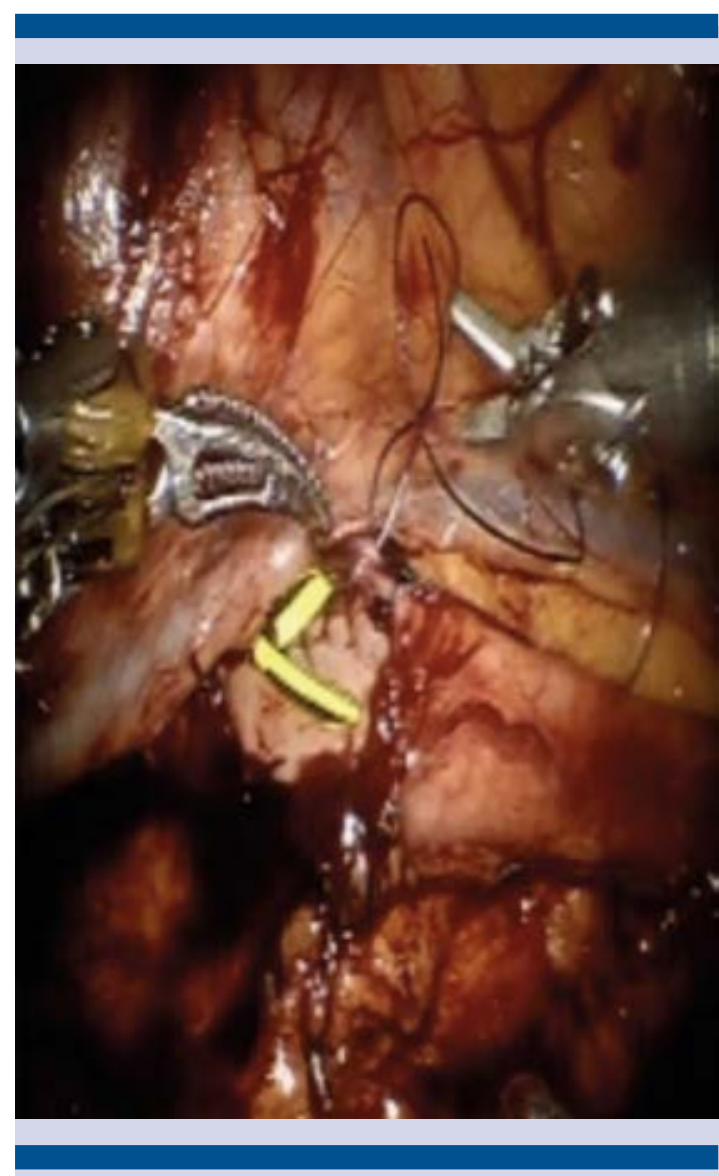

Figura 9. Finalización de la anastomosis.

\section{Conflicto de intereses}

Los autores declaran no tener conflicto de intereses.

\section{REFERENCIAS}

1. Sivaraman A, Leveillee RJ, Patel MB, Chauhan S, et al. Robot-assisted laparoscopic dismembered pyeloplasty for ureteropelvic junction obstruction: a multi-institutional experience. Urology 2012;79(2):351-355.

2. Lee RS, Retik AB, Borer JG, Peter CA. Pediatric robot assisted laparoscopic dismembered pyeloplasty: comparison with a cohort of open surgery. J Urol 2006;175(2):683687.

3. Lucas SM, Sundaram CP, Wolf JS, Leveillee RJ, Bird VG, et al. Factors that impact the outcome of minimally invasive pyeloplasty: results of the Multi-institutional Laparoscopic and Robotic Pyeloplasty Collaborative Group. J Urol 2012;187(2):522-527.

4. Chen RN, Moore RG, Kavoussi LR. Laparoscopic pyeloplasty: Indications, technique, and long-term outcome. Urol Clin North Am 1998;25(2):323-330.

5. Gettman MT, Neururer R, Bartsch G, Peschel R. AndersonHynes dismembered pyeloplasty performed using the da Vinci robotic system. Urology 2002;60:509-13.

6. Binder J, Bräutigam R, Jonas D, Bentas W. Robotic surgery in urology: fact or fantasy?. BJU international 2004;94(8):1183-1187.

7. Sung GT, Gill IS, Hsu TH. Robotic-assisted laparoscopic pyeloplasty: a pilot study. Urology 1999;53:1099.

8. Khan N, Abboudi H, Khan MS, Dasgupta P, Ahmed K. Measuring the surgical 'learning curve': methods, variables and competency. BJU Int 2014;113(3):504-508.

9. Varda BK, Johnson EK, Clark C, Chung BI, et al. National trends of perioperative outcomes and costs for open, laparoscopic and robotic pediatric population. J Urol 2014;191(4):1090-6.

10. Kommu SS, Murphy D, Patel SP. Robot assisted pyeloplasty vs. laparoscopic pyeloplastya-preliminary cost comparison in the United Kingdom setting. J Endourol, 2006;20S1:MP19-04.

11. Mufarrij PW, Woods M, Shah OD, Palese MA, et al. Robotic dismembered pyeloplasty: a 6-year, multi-institutional experience. J Urol 2008;180(4):1391-1396. 